\section{PERCEPCIÓN DEL ALUMNADO SOBRE LA EVALUACIÓN DE HISTORIA EN EDUCACIÓN SECUNDARIA OBLIGATORIA: ANÁLISIS DE LAS CALIFICACIONES ESPERADAS Y OBTENIDAS}

\author{
Francisco Javier Trigueros Cano \\ Universidad de Murcia \\ ORCID iD: https://orcid.org/0000-0002-7098-0927 \\ javiertc@um.es \\ Pedro Miralles Martínez \\ Universidad de Murcia \\ ORCID iD: https://orcid.org/0000-0002-9143-2145 \\ pedromir@um.es \\ Jesús Molina Saorín \\ Universidad de Murcia \\ ORCID iD: https://orcid.org/0000-0002-9919-0910 \\ jesusmol@um.es \\ Antonio Maurandi López \\ Universidad de Murcia \\ ORCID iD: https://orcid.org/0000-0002-4292-8312 \\ amaurandi@um.es
}

Cómo citar este artículo/Citation: Trigueros Cano, F. J., Miralles Martínez, P., Molina Saorín, J. y Maurandi López, A. (2018). Percepción del alumnado sobre la evaluación de historia en Educación Secundaria Obligatoria: análisis de las calificaciones esperadas y obtenidas. Arbor, 194 (788): a449. https://doi. org/10.3989/arbor.2018.788n2009

Recibido: 6 marzo 2015. Aceptado: 8 febrero 2016.

RESUMEN: Esta investigación analiza las posibles fuentes de variación y discrepancias entre la calificación obtenida y la que espera obtener a final de curso el alumnado de Educación Secundaria en Historia, según la información recibida sobre los criterios de evaluación y la práctica de determinados hábitos de estudio. Tras la aplicación de un cuestionario con una muestra superior a 1100 alumnos de once institutos, el alumnado mejor informado tiene una diferencia menor entre la nota esperada y la obtenida; en cambio, entre los que afirman no conocer bien los criterios de evaluación, la diferencia es mayor.

PALABRAS CLAVE: Evaluación; ciencias sociales; alumnado; Educación Secundaria.
STUDENT'S PERCEPTIONS ABOUT EVALUATION OF HISTORY IN SECONDARY EDUCATION: ANALYSIS OF EXPECTED AND OBTAINED GRADES
Copyright: (C) 2018 CSIC. Este es un artículo de acceso abierto distribuido bajo los términos de la licencia de uso y distribución Creative Commons Reconocimiento 4.0 Internacional (CC BY 4.0).

ABSTRACT: This research analyzes the possible sources of variation between the grade that students of high school expect at the end of the course and what they really get, according to information received on the evaluation criteria and the practice of certain study habits. After application of a questionnaire with a sample of more than 1100 students who studied the subject of History, from eleven high schools. The study concludes that better informed students have a smaller difference between expected and obtained grade, whereas the differences are greater in students who consider that they don't know the evaluation criteria well.

KEYWORDS: Assessment; social sciences; students; Secondary Education. 


\section{INTRODUCCIÓN}

La coherencia entre las obligaciones de los alumnos y la práctica educativa debería garantizar que entre el alumnado no hubiera sorpresas sobre las notas que obtienen al finalizar el curso. En ello, cobran especial importancia la actitud del alumnado, la adquisición de unos hábitos de estudio y el conocimiento sobre cómo se les va a valorar. Por tanto, la información que el alumno recibe sobre los criterios de evaluación se convierte en una tarea muy importante para el correcto desarrollo de los procesos de aprendizaje y para conseguir una evaluación positiva. Conviene saber cuándo y cómo se informa a los alumnos sobre estos criterios, cuándo y cómo el alumno dice conocerlos y, por supuesto, la influencia o no de dicha información en la predicción y consecución de la nota final. Del mismo modo, es importante la predisposición del alumnado hacia la materia y la importancia que el profesorado otorgue al conocimiento que el estudiante debe tener sobre los criterios de evaluación. En este trabajo, se analizan las respuestas ofrecidas por el alumnado con relación a este particular. El interés por conocer algo más sobre el pensamiento del colectivo discente, al relacionar las calificaciones obtenidas con los resultados esperados, a tenor de variables como tiempo dedicado al estudio, la importancia de la materia, el tipo de exámenes y contenidos de los mismos, los hábitos de estudio, la información recibida sobre los criterios de evaluación, etc., nos ha llevado a la propuesta de realización de un estudio descriptivo e interpretativo, con metodología cuantitativa, aplicado a alumnos de 3. y 4. de Educación Secundaria Obligatoria (ESO). La información recibida de los agentes implicados en el proceso educativo puede aportar datos de gran valor para entender mejor la realidad de nuestras aulas y comprender los porqués de los resultados académicos en ciencias sociales, así como el grado de corresponsabilidad en los mismos por parte del alumnado. De este modo, será posible enunciar propuestas y cambios, en su caso, para la mejora educativa (Porto, García y Navarro, 2013). En esta línea, autores como McIntyre, Pedder y Rudduck (2005) afirman que la motivación del alumnado y su actitud hacia la materia cambian de forma muy positiva cuando el profesorado tiene en cuenta sus opiniones y sus puntos de vista, pues les hace partícipes y corresponsables de los procesos educativos. Si los alumnos son una parte muy importante del proceso evaluador, será necesario conocer sus opiniones; en este sentido, hay trabajos que demuestran que las respuestas ofrecidas por los alumnos, en relación con la enseñanza y el aprendizaje en las distintas materias, indican que para ellos son muy importantes los procesos internos (Waug, 2009), además de los desarrollos finales. En este contexto surgen una serie de preguntas de investigación: ¿debería coincidir la nota que los alumnos esperan con aquella que obtienen?, ¿la información que recibe el alumnado sobre los criterios de evaluación es relevante en esta cuestión?, ¿en qué consiste y cómo se realiza ese proceso informador?, ¿̇ocupa un papel relevante en la percepción sobre la nota el desarrollo de unos hábitos de estudio? En este estudio se analizan las posibles fuentes de variación entre la calificación que el alumno espera obtener a final de curso (nota esperada o expectativa) y la que realmente termina alcanzando (nota obtenida) en geografía e historia de 3. y 4. de ESO. Coincidimos con Waugh (2009) al afirmar que el alumno tiene un mayor control y mejor relación con sus compañeros si su opinión se considera para la evaluación. Siendo la historia una disciplina en la que el profesor suele dirigir más el proceso evaluador, la consideración y mayor participación del alumnado en su evaluación produce más efectos a largo plazo en los resultados académicos, en comparación con otras materias (matemáticas, por ejemplo). Del mismo modo, conviene destacar que cualquier análisis que se realiza desde el punto de vista del discente está plagado de mitos, creencias y prejuicios que, obviamente influyen en la decisión que adoptan hacia el aprendizaje de cualquier materia (Heine y Maddox, 2009). En esta misma línea, Gentry, Gable y Rizza (2002) consideran que evaluar las clases desde el punto de vista de los estudiantes es importante, ya que las preferencias y perspectivas de los mismos son diferentes a las de sus profesores, lo que nos aportará datos desconocidos por el docente. A pesar de la abundante bibliografía existente sobre la evaluación en general, al ser considerada como una tarea cuya responsabilidad se le atribuye al colectivo docente, en ella no suele tenerse en cuenta la opinión del alumnado. No obstante, hay estudios que resaltan la conveniencia de contar con los estudiantes en este proceso, pues hace que desarrollen una actitud hacia la materia altamente positiva y satisfactoria en estos procesos. Journell (2010) ha investigado sobre la percepción del alumnado en alguna de las ciencias sociales, principalmente en historia y geografía, destacando la importancia de tener en cuenta la opinión del alumno. Heckert, Ltier, Ringwald-Burton y Drazen (2006) afirman que el esfuerzo del estudiante está más relacionado con la evaluación (actitud) del profesor que con la nota esperada. Además, el alumno de ESO agradece que se tenga en cuenta su opinión en esta materia, y al estar bien informado hace que sea más sencillo obtener resultados positivos, al tiempo que facilita los conocimientos. McCall (2006) indica que si el profesor comunica los objetivos 
que hay que conseguir y el propósito de la asignatura, la actitud del alumnado es bastante más positiva. Para otros autores (Molina, Nunes y Vallejo, 2012) la evaluación formativa es un proceso bidireccional entre profesores y alumnos, con el fin de aumentar, reconocer y responder al aprendizaje. Esta es la oportunidad ideal para que los alumnos expresen cuáles son sus expectativas de aprendizaje y, sobre todo, aprendan de qué forma van a ser evaluados (Burns, 2010). Por su parte, Chevalier, Gibbons, Hoskins, Snell y Torpe (2008) indican que los estudiantes deberían ser entrenados para desarrollar una visión de sus objetivos en función de sus habilidades, lo que incidiría en sus expectativas sobre los resultados académicos. Según Heine y Maddox (2009), suele haber correlaciones positivas entre la nota que el estudiante obtiene académicamente y la nota que otorga al profesor; es decir, que los estudiantes que no obtienen buenos resultados evalúan mal al profesorado y a la práctica docente. Maurer (2006) estableció que una variable que afecta a la precisión de las percepciones del estudiante era la disonancia cognitiva. Esto nos lleva a pensar que la esperanza sobre los resultados de los alumnos con malos hábitos no será igual que la de aquellos que tienen buenos hábitos.

\section{MATERIAL Y MÉTODO}

Para la realización de esta investigación, se diseñó un cuestionario denominado Escala de Percepción de la Evaluación en Geografía e Historia (EPEGEHI-1), el cual fue aplicado al alumnado de 3. y 4.ㅇ de ESO de la Región de Murcia antes de que conocieran la nota final del curso, revelándose así las expectativas sobre la nota que esperaban obtener. Para el análisis de los datos, se ha utilizado el paquete estadístico R 2.15 ( $R$ Core Team, 2012) y SPSS. El muestreo total se aplica a once institutos obteniéndose 1.117 encuestas válidas (error muestral: ,7\%). La muestra resulta homogénea en cuanto a sexo (53\% varones, $46 \%$ mujeres). Las edades se distribuyen en tres grandes grupos: $39 \%$ con 15 años, $42 \%$ con 16, junto a un elevado porcentaje de repetidores (23\%). La distribución del alumnado por niveles es la siguiente:

- Estudiantes que cursan 4.․: De ellos se dispone de registros sobre sus tres notas de tercer curso y dos de 4. (1. - y 2. a evaluaciones), además de

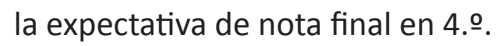

- Alumnado que cursa 3.. : De ellos se dispone de registros sobre las tres notas de segundo curso y dos notas de 3. (evaluaciones 1. - y 2. a $)$, además de su expectativa de nota final en 3.․․
Se han contrastado las notas de las dos primeras evaluaciones junto con la nota final obtenida (por un lado) y la esperada (por otro). Así, se crean tres nuevas para tercer curso: "notas3_1" y "notas3_2", que no tienen valores faltantes; y notas3_3", formada por los sujetos que ahora están en 4. o. Por último, debemos destacar que la variable "expectativa de nota a obtener" se refiere al curso actual, así que para relacionarla con las notas de 3. o solo será válida si tenemos en cuenta los alumnos que ahora están en 3.o. Por tanto, mientras que para "notas3_3" solo teníamos datos de alumnos que están en 4.․, para "expectativa de nota a obtener" solo tenemos registros válidos para los que están en 3.․ Debemos tener presente que, en la actitud del alumnado sobre la nota final, es importante saber qué sabe sobre la forma de calificar la materia, es decir, si conoce los criterios de evaluación y calificación y su aplicación en los procesos de enseñanzaaprendizaje (Tabla 1 ) y el tiempo de estudio o de trabajo que le dedica a la materia de geografía e historia (es decir, los hábitos de estudio y su incidencia en el aprendizaje y evaluación de esta materia (Tabla 2).

Una vez contestadas las cuestiones anteriores, se crea una variable agregada de información sobre evaluación, originándose dos grupos: alumnos que expresan estar bien informados y alumnos que dicen estar mal informados, averiguando además si esa información se recibe al inicio de curso o se produce de forma continuada a lo largo del mismo. Como también se pretende conocer la relación con los hábitos de estudio, en la Tabla 2 se reflejan las preguntas relacionadas con este apartado del estudio:

Estas respuestas permiten crear una nueva variable agregada, denominada "Hábitos de estudio". Para su definición, se consideran alumnos con buenos hábitos aquellos que estudian una hora o más a la semana (recordar que geografía e historia se imparte tres horas por semana) y preparen el examen, como mínimo, el día anterior.

\section{RESULTADOS}

En primer lugar, para analizar los resultados, se elabora una primera clasificación para conocer las notas obtenidas en las distintas evaluaciones, y aquella que esperan obtener en la evaluación final. En la Tabla 3 se muestra un análisis de descriptivos en el que aparece el número de estudiantes que han participado en la investigación (con las notas medias obtenidas y esperadas en las distintas evaluaciones de 3.), distinguiéndose en función del curso en el que están matriculados. Se observan las mayores diferencias (signifi- 
Tabla 1. Preguntas del cuestionario relacionadas con la información que el alumnado recibe sobre los criterios de evaluación

\begin{tabular}{|l|l|l|}
\hline V1 & $\begin{array}{l}\text { A principio de curso, el profesor nos informó de los criterios de calificación de la asignatura } \\
\text { de ciencias sociales, geografía e historia. }\end{array}$ & $\square$ Sí \\
\hline V2 & $\begin{array}{l}\text { ¿Para la evaluación, el profesor tiene en cuenta sobre todo la nota de los exámenes? Es } \\
\text { decir, ċlos exámenes suponen el mayor porcentaje de la calificación final? }\end{array}$ & $\square$ Sí \\
\hline & Además de los exámenes ¿qué otros aspectos tiene en cuenta el profesor para evaluarte? Señala lo que consideres \\
\hline V3 & La participación y las preguntas hechas en clase & $\square$ Sí \\
\hline V4 & El comportamiento y la actitud en clase & $\square$ Sí \\
\hline V5 & $\begin{array}{l}\text { La realización de los ejercicios y/o actividades que manda el profesor y que se hacen dentro } \\
\text { de clase }\end{array}$ & $\square$ Sí \\
\hline V6 & Trabajos, deberes y/o proyectos que hay que hacer fuera de clase & $\square$ Sí \\
\hline & Otros (pon ejemplos): & $\square$ Ní \\
\hline V7 & Antes de hacer el examen, ¿̇te han explicado o recordado cómo se va a calificar? & $\square$ Sí \\
\hline & & $\square$ No \\
\hline V8 & Lo que vale cada pregunta, o lo que puntúa, ¿se especifica claramente en el examen? & $\begin{array}{l}\square \text { Casi siempre } \\
\text { A veces }\end{array}$ \\
\hline
\end{tabular}

Fuente: elaboración propia.

Tabla 2. Preguntas sobre el tiempo de estudio

\begin{tabular}{|l|l|}
\hline \multirow{4}{*}{ V9 } & Para la preparación del examen de la asignatura de ciencias sociales, ¿̇cuándo estudias? \\
\hline & $\square$ Normalmente, me presento al examen sin haber estudiado \\
\hline & $\square$ Estudio únicamente el día o la noche antes del examen \\
\hline & $\square$ Estudio unos días antes del examen \\
\hline & $\square$ Estudio desde la semana antes del examen \\
\hline & $\square$ Estudio semanalmente, desde el inicio del curso \\
\hline & $\square$ Estudio prácticamente a diario, desde el inicio del curso \\
\hline & $\square$ Otra respuesta (especifícala): \\
\hline \multirow{3}{*}{ V10 } & ¿Cuánto tiempo dedicas a la semana a la asignatura de ciencias sociales? \\
\cline { 2 - 3 } & $\square$ Normalmente no estudio $\square$ Menos de 1 hora semanal $\square 1$ hora $\square$ De 1 a 2 horas $\square$ De 2 a 3 horas \\
& $\square$ De 3 a 4 horas $\square$ Más de 4 horas a la semana \\
\hline
\end{tabular}

Fuente: elaboración propia.

cativas, $t(1099)=6,170, p<, 01))$ entre la nota obtenida y la esperada correspondiendo con la última evaluación. Por el contrario, en las dos primeras evaluaciones, las diferencias entre las notas obtenidas por los dos grupos (alumnos actualmente en 30 y los alumnos que ya están en 4으) son mínimas ( $\mathrm{t}(1100)=, 946 \mathrm{p}=, 344$ y $\mathrm{t}(1081)=1,9622, \mathrm{p}=, 05)$.

Tal y como se puede apreciar (Tabla 3), se observa que los alumnos obtuvieron notas medias muy similares en las evaluaciones 1. a y 2. a de tercer curso, tanto ese año como el anterior. No ocurre igual al contrastar las notas obtenidas en la 3. - evaluación y la expectativa de nota para el mismo periodo, ya que el contraste de medias resultó significativo con una variación de casi un punto: de 6,32 a 7,20 (t(1099)=6,170, $\mathrm{p}<, 01$, Intervalo de confianza para la diferencia de (,5999, $1,1599))$. A la vista de estos resultados, se plantea la siguiente cuestión: ¿Por qué esa diferencia entre la nota final (real) y la que espera obtener el alumno? En consonancia con el objeto de este estudio, se agrupa al alumnado en función de su conocimiento de tales criterios; es decir, en función de estar bien informado o mal informado (alumnos con buenos y malos hábi- 
Tabla 3. Nota media obtenida en ciencias sociales en 3. de ESO

\begin{tabular}{|c|c|c|c|c|}
\hline & NOTAS & $\mathbf{N}$ & MEDIA & DESV. TÍPICA \\
\hline \multirow{2}{*}{ 1.a evaluación } & Nota obtenida (alumnado de 3. ㅇ) & 623 & 6,69 & 1,78 \\
\hline & Nota obtenida (alumnado de 4. o) & 479 & 6,79 & 1,72 \\
\hline \multirow{2}{*}{ 2. a evaluación } & Nota obtenida (alumnado de 3. o) & 614 & 6,81 & 1,85 \\
\hline & Nota obtenida (alumnado de 4. o) & 469 & 7,02 & 1,70 \\
\hline \multirow{2}{*}{ 3. a evaluación } & Nota esperada (alumnado de 3. ․) & 630 & 6,32 & 2,72 \\
\hline & Nota obtenida (alumnado de 4. ㅇ) & 471 & 7,20 & 1,71 \\
\hline
\end{tabular}

Fuente: elaboración propia.

tos de estudio (Figura 1). Concretamente, se intenta contrastar si los resultados de los alumnos que durante ese año están cursando 3. y los del año anterior son comparables en las evaluaciones 1. a y 2. a. Es decir, si sus notas no difieren estadísticamente en dichas evaluaciones. Para esta tarea, se ha aplicado una prueba $t$ de Student para el contraste de medias de las variables notas 3_1 y notas 3_2 entre los alumnos de 3. y 4., obteniendo resultados no significativos en ambos casos $(t(1100)=, 946 p=, 344$ y $t(1081)=1,9622$, $p=, 05$, con un tamaño del efecto $r=, 06$ ínfimo). Al contrario, al comparar la nota obtenida en la 3. $\underline{\text { a evalua- }}$ ción de los alumnos de 4.9 y la nota esperada en la misma evaluación por los alumnos que están en 3.ㅇ, observamos diferencias significativas $(t(1099)=6,170$, $\mathrm{p}<, 01)$ con un intervalo de confianza del $95 \%$ para la diferencia entre expectativa y nota obtenida (,5999, $1,1599)$ y una estimación puntual de, 8797 .

De esta manera, se plantea la posibilidad de explicar esta discrepancia teniendo en cuenta la información que dice recibir el alumno sobre los criterios de evaluación, para lo cual adquieren especial importancia los siguientes aspectos:

- Información que el discente recibe a principio de curso sobre los criterios de calificación de la asignatura.

- Averiguar si la nota del examen es lo que más puntúa en la evaluación final.

Figura 1. Análisis de discrepancia entre la expectativa sobre la nota a obtener y la obtenida según el criterio de información (denotamos con $\Delta$ la estimación puntual de la diferencia)

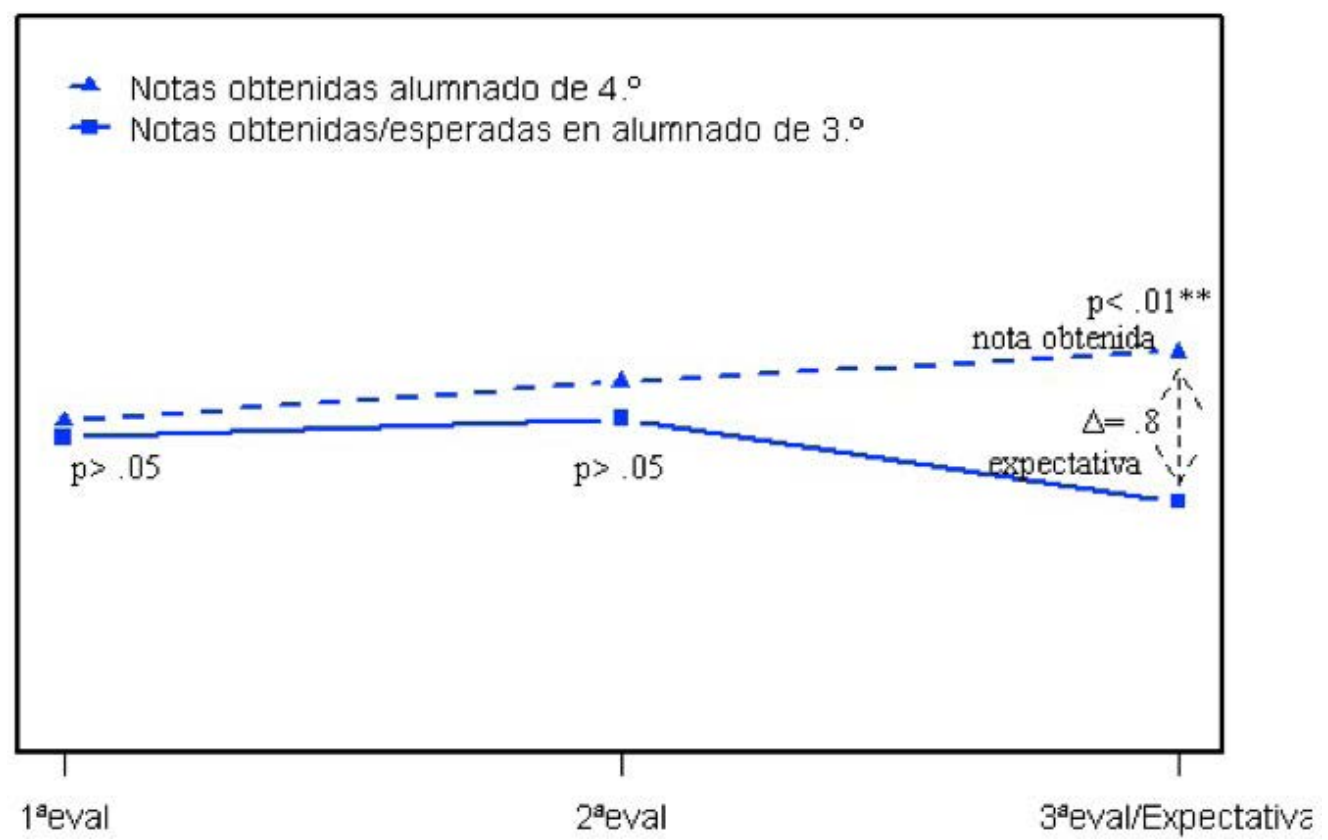

Fuente: elaboración propia. 
- Otros aspectos (además del examen) que el profesor pueda tener en cuenta a la hora de evaluar:

- Participación y preguntas de clase.

- Comportamiento y actitud en clase.

- Realización de las actividades que propone el profesor en clase.

- Trabajos y deberes fuera del aula.

- Otros.

Conocer los criterios de calificación de cada examen, con una clara especificación sobre lo que puntúa cada una de las preguntas de la prueba.

Por tanto, ¿cuántos alumnos expresan que el profesor les informa al inicio del curso sobre los criterios de evaluación? Es decir, ¿cuántos contestan afirmativamente a la pregunta: "A principio de curso, el profesor nos informó de los criterios de calificación de la asignatura de geografía e historia"? La respuesta es abrumadora: el $94 \%$ indica que el profesor sí informa (Tabla 4).

En el segundo caso, para tener una información más rigurosa y completa sobre los datos que el alumnado maneja en relación con los criterios de evaluación, se quiere conocer, además, cómo es esta información que el alumno dice recibir, cómo se plasma, en qué consiste y qué apartados incluye; para esto, trabajamos con las variables del cuestionario (v1, v2, v3, v4, v5 y v6; y v7 y v8). La información procedente de estas variables se organiza en otra nueva, denominada $v a$ riable de información ( $v \_i n f o$ ). Esta nos permite clasificar a los alumnos bien o mal informados con mayor rigor; ya no es válido que contesten afirmativamente a una sola pregunta; en este caso, los requisitos para considerar a un estudiante bien informado son los siguientes: si contesta afirmativamente a la variable v1 (puesto que únicamente admite las respuestas sí o no); que el sumatorio de v2, v3, v4, v5 y v6 sea mayor que 2 (prácticamente, todas las respuestas deben ser afirmativas) y, además, las variables $v 7 \mathrm{y}$ v8 deben ser igual o mayor que 3 (casi siempre). Se trata de un criterio bastante riguroso, pues obliga al profesor a informar al inicio de curso sobre el valor de cada pregunta o apartado y, sobre todo, antes de la realización de los exámenes. En este caso, se obtiene un porcentaje muy bajo de alumnos que dicen estar bien informados de forma continua, es decir, a lo largo de todo el curso: solo un $15,5 \%$ dice conocer bien los criterios de evaluación. Es de destacar el elevado número de alumnos que dice no conocer de forma continuada el valor de los distintos apartados para la elaboración de la nota final $(84,5 \%)$. ¿Por qué esta discrepancia? ¿Acaso el profesor piensa que únicamente indicándolo al inicio del curso el alumnado ya ha asimilado y entendido lo que tiene que hacer para superar la materia? Las categorías de alumnos bien y mal informados (según el criterio anterior) nos llevan a la reflexión de que quizá se haya sido demasiado exigentes y rigurosos al definir los parámetros que clasifiquen al alumno bien o mal informado a lo largo de todo el curso o de forma continuada, por lo que decidimos no exigir nada con respecto a las variables v7 y v8. A la hora de definir un nuevo criterio de información menos restrictivo ("Antes de hacer el examen, ¿te han explicado o recordado cómo se va a calificar?" y "Lo que vale cada pregunta, o lo que puntúa, ¿se especifica claramente en el examen?"). Para ordenar los resultados según estas variables, se crea la variable $v \_$info 20 "Criterio de información sin tener en cuenta $v 7$ y $v 8$ ". Este es menos riguroso que el anterior, pues no obliga al profesor a indicar cómo se va a puntuar cada pregunta o parte de una prueba o examen, pero sí se tiene en cuenta la información que el alumno recibe al inicio del curso, fundamentalmente. El resultado en esta ocasión cambia sustancialmente, pues se obtiene un $98,4 \%$ de alumnos que reconocen que a principio de curso el profesor les informa de los

Tabla 4. A principio de curso el profesor informa de los criterios de calificación

\begin{tabular}{|l|c|c|}
\hline & Frecuencia & Porcentaje \\
\hline sí & 1050 & 94,0 \\
\hline NO & 60 & 5,4 \\
\hline Total & 1110 & 99,4 \\
\hline Perdidos & 7 & 0,6 \\
\hline Total & 1117 & 100,0 \\
\hline
\end{tabular}

Fuente: elaboración propia. 
criterios de evaluación (v1 hasta v6), alguno más que los de la pregunta directa v1 (94\%). Por ello, se deduce que a principio de curso se informa al alumnado de forma general de los procedimientos y criterios de evaluación. Esta información no se ofrece con el mismo rigor a lo largo de todo el curso, de una manera más continua o permanente, pues el alumnado, en su inmensa mayoría, manifiesta no haber sido informado sobre aspectos como el valor de cada examen en el cómputo total de la evaluación, el valor de cada pregunta o apartado del examen, etc. (Tabla 5).

Según la información anterior, volvemos a una de nuestras cuestiones cruciales, cinfluye en la nota que obtiene o espera el alumno la información que recibe sobre los criterios de evaluación? En la Tabla 6 se aprecian las diferencias entre alumnos bien y mal informados, y la nota esperada y obtenida y que, efectivamente, la información que el alumno posee sobre los criterios de evaluación es un factor determinante en la esperanza sobre las notas que espera conseguir, pues se observa casi un punto de diferencia entre lo que espera obtener y aquello que finalmente obtiene entre los alumnos mal informados, de 6,31 a 7,26 ( $t(927)=6,077, p=, 000<, 01)$ y de menor valor entre los bien informados, de 6,38 a 6,96 (t(170)=1,581, $p=, 116)$.

Por otra parte, se pretende averiguar si las diferencias observadas en las notas obtenidas entre los alumnos bien y mal informados son estadísticamente significativas. Para esto se procede a contrastar los resultados mediante una $t$ de Student con las notas esperadas y obtenidas para los dos grupos. El resultado revela que se mantiene la discrepancia únicamente para los alumnos mal informados. Para los alumnos bien informados (Figura 2) encontramos que no hay diferencias significativas entre expectativa y nota real para la 3. a eval. (t $(170)=1,581, p=, 116)$. Sin embargo, para los alumnos mal informados sí encontramos diferencias significativas entre expectativa y nota obtenida en la 3. a evaluación (t (927) $=6,077, p=, 000<, 01$ ), con un intervalo de confianza al 95\% para la diferencia entre expectativa y nota real $(, 644,1,258)$ y una estimación puntual de, 9511 .

Se puede concluir afirmando que los estudiantes bien informados, en su previsión entre lo que esperan y obtienen, casi siempre aciertan; en cambio, los mal informados suelen obtener mayor nota de la que esperan, quizá por no conocer adecuadamente los criterios de información; es decir suelen ser más pesimistas en relación con el rendimiento académico. Como se ha indicado, también se pretende averiguar la relación entre nota esperada y obtenida en relación con los hábitos de estudio. Así, teniendo en cuenta los parámetros anteriores para identificar a los alumnos con buenos o malos hábitos, se obtiene una clasificación en la que se observa con mucha claridad que un

Tabla 5. Criterio de información. Variable de información

\begin{tabular}{|c|c|c|}
\hline Criterio & $\begin{array}{c}\text { V_info2: Criterio de información sin } \\
\text { tener en cuenta v7 y v8 }\end{array}$ & V_info: Criterio de información \\
\hline Alumnado & Porcentaje (Frecuencia) & Porcentaje (Frecuencia) \\
\hline Expresa NO estar bien informado & $1,6 \quad(18)$ & $84,5 \quad(944)$ \\
\hline Dice Sí estar bien informado & 98,4 (1099) & $15,5 \quad(173)$ \\
\hline Total & $100 \quad(1117)$ & $100 \quad$ (1117) \\
\hline
\end{tabular}

Fuente: elaboración propia.

Tabla 6. Nota media esperada y obtenida según criterio de información

\begin{tabular}{|c|c|c|c|c|}
\hline & & $\mathbf{N}$ & MEDIA & DESVIACIÓN TÍPICA \\
\hline \multirow{2}{*}{ Mal informados } & Nota esperada (alumnado de 3. ) & 556 & 6,31 & 2,69 \\
\hline & Nota obtenida (alumnado de 4. ㅇ) & 373 & 7,26 & 1,67 \\
\hline \multirow{2}{*}{ Bien informados } & Nota esperada (alumnado de 3. ) & 74 & 6,38 & 2,92 \\
\hline & Nota obtenida (alumnado de 4. ㅇ) & 98 & 6,96 & 1,82 \\
\hline
\end{tabular}

Fuente: elaboración propia. 
Figura 2. Relación entre notas, expectativas de notas y criterios de información en la 3.a evaluación del curso 3.은

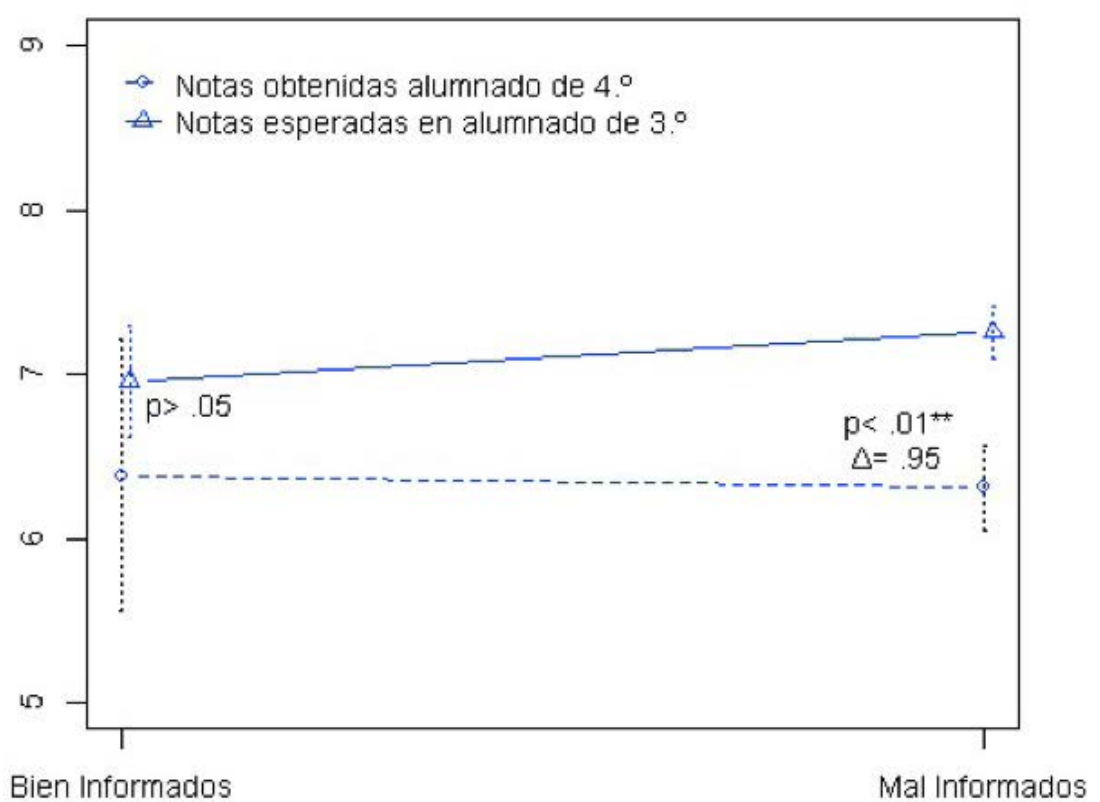

Fuente: elaboración propia.

$77,6 \%$ no tiene hábitos de estudio adecuados y solo un $22,4 \%$ puede considerar que tiene buenos hábitos (Tabla 7).

En la Tabla 8 se presentan varios datos entre alumnos con buenos y malos hábitos, mostrando sus expectativas sobre la nota y aquella que realmente obtienen.

Para los alumnos con buenos hábitos de estudio encontramos que sí hay diferencias significativas entre expectativa y nota real para la 3.a evaluación $(t(807)=6,131, p=, 000<, 05)$, con un intervalo de confianza del $95 \%$ para la diferencia entre expectativa y nota real $(710,1,322)$ y una estimación puntual de 1,016 (Figura 3). Para los alumnos con malos hábitos encontramos que no hay diferencias significativas entre expectativa y nota real para la 3. a evaluación $(t(290)=1,733, p=, 084)$.

Por tanto, se deduce que los alumnos con buenos hábitos obtienen significativamente notas superiores de las que esperan, mientras que los alumnos con malos hábitos no difieren en su percepción entre la nota esperada y la obtenida. Esto podría reforzar la idea de que los buenos hábitos traen consecuencias positivas inmediatas, pues este alumnado es más exigente consigo mismo.

\section{DISCUSIÓN Y CONCLUSIONES}

Según los resultados de este trabajo, el alumnado no conoce -con claridad- cómo se le va a evaluar, pues las respuestas que ofrece para identificar si conoce los criterios de forma global, por apartados, inicial o continuadamente, ofrecen resultados distintos. Hay respuestas que discrepan según sea la pregunta, aunque el contenido sea el mismo; así, cuesta trabajo entender por qué el $94 \%$ de los consultados dice saber los criterios de evaluación al inicio del curso, y cuando esta pregunta no se le hace de forma general, sino que se divide entre los distintos elementos que influyen en la

Tabla 7. Hábitos de estudio

\begin{tabular}{|c|c|c|c|}
\hline \multicolumn{2}{|c|}{} & FRECUENCIA & PORCENTAJE \\
\hline Válidos & Buenos hábitos & 250 & 22,4 \\
\hline & Malos hábitos & 867 & 77,6 \\
\hline & Total & 1117 & 100,0 \\
\hline
\end{tabular}

Fuente: elaboración propia. 
Tabla 8. Notas medias 3. a evaluación según hábitos de estudio

\begin{tabular}{|c|c|c|}
\hline & MALOS HÁBITOS & BUENOS HÁBITOS \\
\hline NOTA ESPERADA (alumnado de 3.) & $\begin{array}{c}6,27 \\
(\mathrm{~N}=171)\end{array}$ & $\begin{array}{c}6,33 \\
(\mathrm{~N}=459)\end{array}$ \\
\hline & $\mathrm{DT}: 2,68$ & DT: 2,74 \\
\hline NOTA OBTENIDA (alumnado de 4.ํ) & $\begin{array}{c}6,76 \\
(\mathrm{~N}=121)\end{array}$ & $\begin{array}{c}7,35 \\
(\mathrm{~N}=350)\end{array}$ \\
\hline & $\mathrm{DT}: 1,75$ & DT: 1,66 \\
\hline
\end{tabular}

$\mathrm{N}$ indica el número de sujetos. DT indica la desviación típica

Fuente: elaboración propia.

Figura 3. Relación entre notas y expectativas de notas y hábitos del alumnado en la 3. a evaluación del curso 3.을

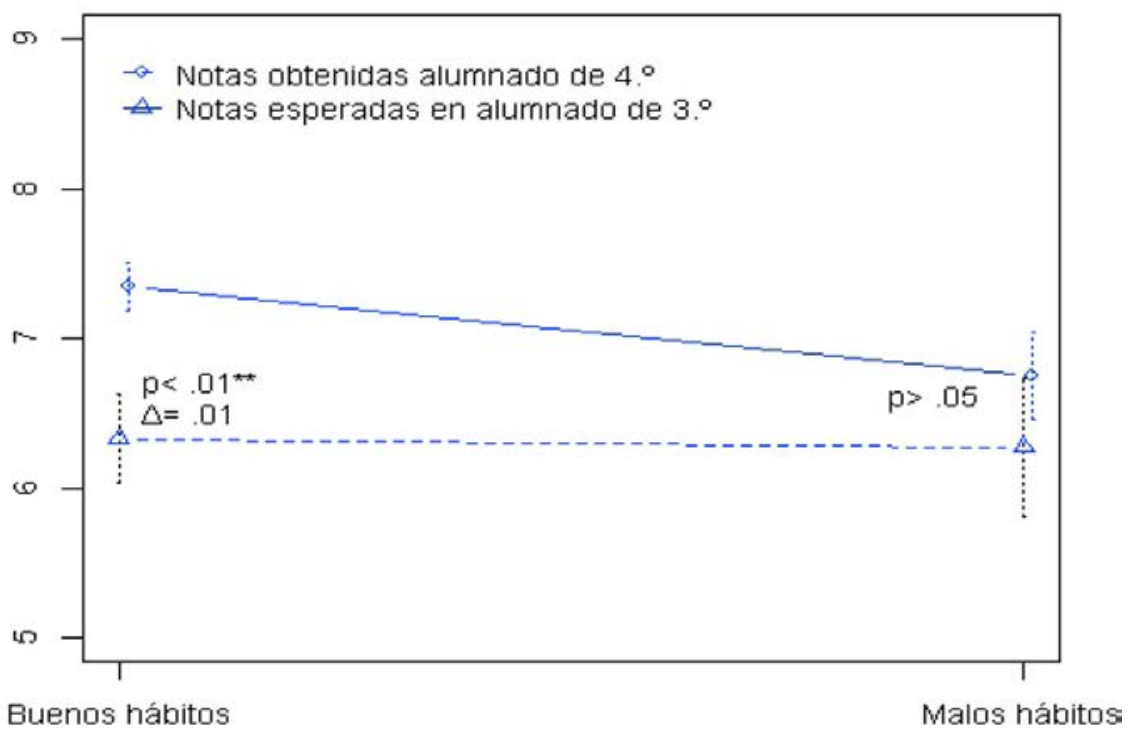

Fuente: elaboración propia.

evaluación, el grupo que afirma conocer estos criterios aumenta hasta el 98,4\%. Ello nos muestra que al pormenorizar o aclarar el contenido de las preguntas, el alumnado entiende mejor lo que el profesor le indica; es como si accediese mejor a esa información cuando se le pregunta si sabe que tal criterio vale más o menos o si el profesor le ha informado que también se tendrá en cuenta otros aspectos además del examen. Teniendo en consideración los estudios de Chapin (2006), así como los de Zhao y Hoge (2005), la materia de historia aparece como la que menos gusta y está considerada de menor utilidad, según los alumnos; en este estudio estas conclusiones se pueden ver reflejadas en el gran número de estudiantes que no tienen buenos hábitos de estudio, pues si la materia les aburre o no les gusta, no le dedican el tiempo suficiente, lo que también influye en el pesimismo que suelen mostrar a la hora de predecir la nota, pues no se ha visto que se trate de estudiantes que prevean alcanzar calificaciones superiores a las obtenidas.

Por otro lado, teniendo en cuenta estudios como los de Mclntyre et al. (2005) y Waugh (2009), la actitud del estudiante ante la materia cambia positivamente cuando se siente partícipe y corresponsable del proceso educativo; o los estudios de Burns (2010), al afirmar que la evaluación formativa es un proceso bidireccional entre profesores y alumnos, con el fin de aumentar, reconocer y responder al aprendizaje; también se desprende de nuestro estudio que, como parte de este proceso educacional, conocer de primera mano lo que nuestros alumnos saben sobre todos los aspectos que intervienen en la evaluación a lo largo del curso es un elemento de vital importancia y que incide directamente en la opinión que el alumno tie- 
ne sobre la nota que espera obtener. Quizás se echen en falta esos diálogos entre profesor-alumno para argumentar (el primero) y expresar sus dificultades (el segundo), al igual que cuando se expone cualquier contenido y después se le dice al estudiante que lo explique con sus propias palabras o que ponga algún ejemplo que, realmente, demuestre que eso se ha entendido. Siguiendo a Waug (2009), trabajar teniendo en cuenta la visión del estudiante sobre la evaluación es altamente positivo, además de ayudar en los procesos de enseñanza y aprendizaje. Si esto es positivo para la enseñanza, ¿el profesorado intenta conocer lo que el alumnado sabe sobre la evaluación?, ¿’por qué esa desorientación en el desconocimiento de los criterios de evaluación a lo largo del curso por parte del alumnado? Esto abre una gran brecha entre lo que el profesor cree que hace (o dice) y lo que el alumnado asimila o entiende, perjudicando al estudiante al sentirse un sujeto pasivo. La mejora de las prácticas evaluadoras en el aula es muy positiva si tenemos en cuenta la opinión de los alumnos (Pedder y Mclntyre, 2006). En este estudio, consideramos que los alumnos podrían haber acertado más en sus expectativas a partir de la motivación que produce conocer los factores que intervienen en el proceso evaluador; es decir, que para la motivación del alumnado sobre una asignatura y las tareas para obtener una evaluación positiva, se manifiesta como relevante el conocer su opinión y que el profesor se interese por saber qué opina el alumno y qué conoce sobre lo que hay que hacer durante el curso. Cuanto más se trabaja con las impresiones de los estudiantes, más coincidencia hay en las expectativas del alumnado en relación con los procesos de evaluación para el aprendizaje (Waug, 2009). También en este caso, se ha visto que un alto porcentaje de alumnos $(84,5 \%)$ dice no tener una información continuada sobre la forma de evaluar por parte del profesor a lo largo de todo el curso, lo que está directamente relacionado con un mayor error en la previsión sobre los resultados que desea obtener; de ahí la necesidad de conocer lo que el alumno espera de la materia y explicar los procedimientos o técnicas para que esa esperanza se modifique a mejor o sirva para crear certidumbres en los discentes.

En historia, el alumno manifiesta su dificultad no tanto en el desconocimiento de la materia, como en saber el mundo en el que ha de estructurarla para las exigencias evaluadoras; es decir, si relacionamos esto con la participación, ¿sabe un adolescente lo que es participar?, ¿o cuánto ha de participar para que se tenga en cuenta al máximo en su puntuación?, ¿distingue bien y distribuye los espacios y tiempos según la importancia de cada aspecto que hay que tener en cuenta en la evaluación? Lógicamente, estos apartados difíciles de cuantificar pueden producir desorientación e influir en una previsión equívoca de sus posibilidades. Por otro lado, si el alumnado, en los últimos días de curso, muestra dudas sobre la nota que ha de obtener y no espera resultados muy buenos (en general), habrá que incidir en la necesidad de ofrecer la información de forma continua y lo más clara posible. En este caso, coincidimos con Heine y Maddox (2009) cuando afirman que existe una correlación positiva entre notas obtenidas y el grado de información que posee el alumno. Otro aspecto importante que se deduce de este trabajo es que la práctica de unos buenos hábitos de estudio permite obtener puntuaciones mayores en la materia ( 7,35 frente a 6,76 de los malos hábitos), aunque sus expectativas iniciales sean bastante inferiores a las notas reales. Aún teniendo este desfase importante entre lo que se espera y lo que se obtiene, los alumnos con buenos hábitos suelen tener una previsión de la calificación que siempre es más alta $(6,33)$ que la que manifiestan los alumnos con malos hábitos $(6,27)$, aunque al conocerse las puntuaciones haya menos diferencias entre el colectivo con peores hábitos de estudio. Como se ha dicho anteriormente, tal vez se deba al esfuerzo y exigencia propia de los alumnos con buenos hábitos. El elevado número de alumnos con malos hábitos $(77,6 \%)$ nos podría llevar a relacionar este dato con otros factores que intervienen en la educación o con consecuencias como el fracaso escolar. Esta mayoritaria práctica sobre la materia no lleva a la desorientación e inseguridad propias de no dedicar el tiempo necesario al estudio. Por consiguiente, el alumnado que conoce mejor en qué consiste la evaluación y pone en práctica sus obligaciones, tiene mejores expectativas sobre sus resultados académicos. Coincidimos con Chevalier et al. (2008) en ese reconocimiento por parte del alumnado de sus posibilidades académicas. Esta necesidad que debe tener el profesorado sobre lo que el alumnado conoce de la materia, de la evaluación, de las explicaciones, de la estructura de las clases, de sus obligaciones como estudiante, etc. permitirá que, a la hora de adoptar las serias y responsables decisiones sobre una evaluación lo más completa posible (o como últimamente se ha denominado: evaluación auténtica), tenga más elementos y criterios para llevarla a cabo con objetividad. Esto también se refleja en trabajos como los realizados por Mclntyre et al. (2005) cuando afirman que, al preguntar a los estudiantes por los distintos procesos educativos, nos aportan datos y detalles de los procesos internos, y no solo de lo 
que se ve. Tal y como se observa en los resultados, los estudiantes bien informados, en su previsión entre lo que esperan y obtienen, suelen acertar más, es decir, la nota que esperan obtener tiene un mayor grado de coincidencia con la nota que obtienen; y los mal informados, en cambio, tienen menores posibilidades de predecir sus resultados con éxito, e incluso encontrándose en los últimos días del curso suelen manifestar una actitud más pesimista frente a la nota final. Quizá haya que replantearse cómo ha de ser esa información, que por ley el docente ha de ofrecer al alumno sobre los criterios de evaluación. No se trata de comunicarlo durante los primeros días de clase, pues el curso es largo y habrá aspectos que no se entiendan adecuadamente hasta que no se lleven a la práctica. Tampoco es operativo colocar la información en cualquier lugar y pensar que el alumno la consultará cuando le sea necesario. Como se ha expresado antes, a principio de curso se informa al alumnado de forma general de los procedimientos y criterios de evaluación, pero esta información no se ofrece con el mismo rigor a lo largo de todo el curso de una manera continuada. Será el día a día lo que facilite a los estudiantes una predicción de nota con el menor índice de error posible y a partir de su implicación en el proceso educativo. En este punto se observa una relación muy elevada con la evaluación formativa o procesual, tan importante en el proceso de enseñanza-aprendizaje y tan demandada en los últimos años.

\section{AGRADECIMIENTOS}

Este trabajo es resultado de los proyectos de investigación Criterios, procedimientos e instrumentos de evaluación de los contenidos de Geografía e Historia en el segundo ciclo de Educación Secundaria Obligatoria (08668/PHCS/08), financiado por la Fundación Séneca-Agencia de Ciencia y Tecnología de la Región de Murcia en el marco del II PCTRM, y La evaluación de las competencias básicas en Educación Secundaria Obligatoria desde las ciencias sociales (EDU201237909-C03-03), subvencionado por el Ministerio de Economía y Competitividad.

\section{BIBLIOGRAFÍA}

Burns, R. (2010). Implementation of Formative Assessment Strategies as Perceived by High School Students and Teachers: Professional Development Implications. [En línea]. Disponible en http:// scholarsarchive.jwu.edu/dissertations/ AAI3398377/

Chapin, J. (2006). Introduction: Do elementary school students and their teachers really dislike social studies? The Social Studies, 97 (5), pp. 187-188. https://doi. org/10.3200/TSSS.97.5.187-188

Chevalier, A., Gibbons, S., Hoskins, S., Snell, M. y Torpe, A. (2009). Students' Academic Self-Perception. Economics of Education Review, 28 (6), pp. 716-727. https://doi.org/10.1016/j.econedurev.2009.06.007

Gentry, M., Gable, R. K. y Rizza, R. K. (2002). Students' perceptions of classroom activities: Are there grade-level and gender differences? Journal of Educational Psychology, 94, pp. 539-544. https:// doi.org/10.1037/0022-0663.94.3.539

Heckert, T. M., Latier, A., Ringwald-Burton A. y Drazen, C. (2006). Relations among student effort, perceived class difficulty appropriateness, and student evaluations of teaching: Is it possible to "buy" better evaluations through lenient granding? College Student Journal, 40 (3), pp. 588-596.
Heine, P. y Maddox, N. (2009). Student Perceptions of the Faculty Course Evaluation Process: An exploratory Study of Gender and Class Differences. Research in Higher Education Journal, 3, pp. 1-10.

Journell, W. (2010). Perceptions of elearning in secondary education: a viable alternative to classroom instruction or a way to bypass engaged learning? Educational Media International, 47 (1), pp. 69-81. https://doi. org/10.1080/09523981003654985

Maurer, T. (2006). Cognitive dissonance or revenge? Student grades and course evaluations. Teaching of Psychology, 33 (3), pp. 176-179. https://doi. org/10.1207/s15328023top3303_4

McCall, A. (2006). Supporting exemplary social studies teaching in elementary schools. The Social Studies, 97 (4), pp. 161-167. https://doi.org/10.3200/ TSSS.97.4.161-167

Mclntyre, D. Pedder, D. y Rudduck, J. (2005). Pupil voice: comfortable and uncomfortable learnings for teachers. Research Papers in Education 20 (2), pp. 149-168. https://doi. org/10.1080/02671520500077970

Molina, J, Nunes, R. M. y Vallejo, M. (2012). The social perception of people with Down syndrome: the EPSD-1 scale.
Educação e Pesquisa, 38 (4), pp. 949964. https://doi.org/10.1590/S151797022012000400011

Pedder, D. y McIntyre, D. (2006). Pupil consultation: the importance of social capital. Educational Review, 58 (2), pp. 145-157. https:// doi.org/10.1080/00131910600584009

Porto, M., García, M. y Navarro, E. (2013). ¿Qué evalúan los instrumentos de evaluación? Valoraciones de estudiantes. Arbor, 189 (760), a018. https://doi. org/10.3989/arbor.2013.760n2004

R Core Team (2012). R: A language and Environment for Statistical Computing. Vienna: R Foundation for Statistical Computing.

Waugh, C. (2009). Enhancing our understanding of Assessment for Learning: how teachers' use student consultation strategies to inform the development of classroom assessment practices. En: Munn, P. (ed.). British Educational Research Association Annual Conference. Manchester: University of Manchester, pp. 1-17. Disponible en http://www.leeds.ac.uk/educol/documents/187607.pdf

Zhao, Y. y Hoge, J. (2005). What elementary students and teachers say about social studies. The Social Studies, 96 (5), pp. 216-221. https://doi.org/10.3200/ TSSS.96.5.216-221 